\title{
DAISIE and arthropod invasions in Europe
}

\author{
Philip E. Hulme', David B. Roy² \\ I The Bio-Protection Research Centre, Lincoln University, Christchurch, New Zealand 2 Centre for Ecology \& \\ Hydrology, Crowmarsh Gifford, Wallingford, United Kingdom \\ Corresponding authors: Philip E. Hulme (philip.hulme@lincoln.ac.nz),David B.Roy (dbr@ceh.ac.uk)
}

Academic editor: Alain Roques | Received 21 January 2010 | Accepted 18 May 2010 | Published 6 July 2010

Citation: Hulme PE, Roy DB (2010) DAISIE and arthropod invasions in Europe. In: Roques A et al. (Eds) Alien terrestrial arthropods of Europe. BioRisk 4(1): 1-3. doi: 10.3897/biorisk.4.41

A milestone in the knowledge of alien species in Europe has been achieved by the DAISIE (Delivering Alien Invasive Species Inventories for Europe) project. Through the Sixth Framework Programme of the European Union, DAISIE has delivered a major portal for information on biological invasions that is publicly available at http://www. europe-aliens.org. The rationale was to develop a pan-European inventory of invasive alien species by integrating existing databases, to describe patterns and evaluate trends in biological invasions in Europe, identify priority species and assess their ecological, economic and health risks and impacts. Although an on-going process, the foundation, scope, and technological architecture of DAISIE was established through a consortium of leading researchers of biological invasions in Europe from 19 institutions across 15 countries and delivered through the cooperation of experts in ecology and taxonomy from throughout Europe that in total amounted to 182 contributors. The inventory, accounts, and distribution maps today provide the first qualified reference system on invasive alien species for the European region. The information presents an outstanding resource to synthesise current knowledge and trends in biological invasions in Europe. The data will help identify the scale and spatial pattern of invasive alien species in Europe, understand the environmental, social, economic and other factors involved in invasions, and can be used as a framework for considering indicators for early warning.

A key component of DAISIE is The European Alien Species Database, an inventory of all alien species in Europe, and resulted from compiling and peer-reviewing national and regional lists of alien fungi, bryophytes, vascular plants, invertebrates, fish, amphibians, reptiles, birds and mammals. Data were collated for all 27 European Union member states (and separately for their significant island regions), other European states (Andorra, Iceland, Liechtenstein, Moldova, Monaco, Norway, the European

Copyright P.E. Hulme, D.B. Roy. This is an open access article distributed under the terms of the Creative Commons Attribution License, which permits unrestricted use, distribution, and reproduction in any medium, provided the original author and source are credited. 
part of Russia, Switzerland, Ukraine, former Yugoslavia states) and Israel. Marine lists are referenced to the appropriate political region with administrative responsibility. To have full coverage of the European marine area, the data for countries bordering the Mediterranean Sea in North African and Near East countries are included. By November 2008, records of 10,771 alien species, were included in the database, belonging to 4492 genera and 1267 families. Both species of exotic origin and species of European origin introduced in European regions outside their native range were considered. Plants are most represented accounting for $55 \%$ of all taxa (5789 species), terrestrial invertebrates $23 \%$ (2477 species), followed by vertebrates (6\%), fungi $(5 \%)$, molluscs (4\%), Annelida (1\%) and Rhodophyta (1\%). In total, the database includes records of 45,211 introduction events to particular regions (plants: 28,093; terrestrial invertebrates: 11,776; aquatic marine species: 2777 , terrestrial vertebrates: 1478 ; aquatic inland species: 1087). Due to unprecedentedly thorough assessment, DAISIE substantially improved the accuracy of estimates of alien species numbers derived from previous datasets.

The information accumulated by DAISIE has been summarized in the Handbook of Alien Species in Europe (DAISIE 2009), which contains analytical chapters on each taxonomic group, and fact sheets of the 100 most invasive alien species in Europe with distribution maps and images. The book also lists all alien species recorded, ranked taxonomically; this list can be used as a reference for future assessment of trends in biological invasions in Europe. The current volume "Alien terrestrial arthropods of Europe" largely follows the lead set by the Handbook of Alien Species in Europe but provides much needed detail on one of the largest and most complex taxonomic groups, the arthropods.

Unlike other groups of animals and plants, no checklist of alien terrestrial invertebrates was available in any of the European countries until the beginning of this century. Thus more than any other taxonomic group, creating an inventory of invasive alien arthropods in Europe proved to be a major challenge. Consequently, an estimate of the importance of terrestrial alien invertebrates at the European level remained impossible, largely due to the limited taxonomic knowledge regarding several major arthropod groups. As a result, the initial analyses in DAISIE were drawn from the most reliably studies group, the insects. Even with such a partial picture, the new evidence emphasised the need for more detailed assessment of alien arthropods. For example, the initial work in DAISIE has shown that approximately $90 \%$ of terrestrial insects having arrived into Europe unintentionally (75\% associated with a commodity, 15\% as stowaways). The highest numbers of insects occur in human-made habitats (ruderal, cultivated land, parks and gardens) and invasions are concentrated to these few highly invaded habitats. Not surprisingly insects are one of the taxonomic groups with the most species causing impacts in Europe, and most of these impacts are on the economic rather than environmental sectors. In this regard, Alien terrestrial arthropods of Europe extends the initial work in DAISIE and develops a clearer picture of arthropod invasions across a much larger taxonomic range than insects. This substantial work will set the benchmark for authoritative assessments of invasive terrestrial invertebrates. 
Through DAISIE, Europe is today the continent with the most complete information on its alien biota. The continent has been working towards implementing an effective strategy on invasive alien species and DAISIE is considered as one of the major instruments towards achieving this goal. An internet-accessible knowledge base, such as DAISIE, can provide crucial information for the early detection, eradication, and containment of invasive aliens - which is most achievable for species that have just arrived. As a result of DAISIE, managers and policy-makers addressing the invasive alien species challenge can easily obtain data on which species are invasive or potentially invasive in particular habitats, and use this information in their planning efforts. Agencies responsible for pest control can quickly determine if a species of interest has been invasive elsewhere in Europe. Importers of new alien species can access data to make responsible business choices. Land managers can learn about control methods that have been useful in other areas, reducing the need to commit resources for experimentation and increasing the speed at which control efforts can begin. DAISIE is potentially a model for other continents which currently have much less detailed information on their alien biota.

\section{References}

DAISIE (2009) Handbook of Alien Species in Europe. Dordrecht: Springer. 